\title{
Anxiolytic and neuroprotective effects of the Traditional Chinese Medicinal formulation Dan-zhi-xiao-yao-san in a rat model of chronic stress
}

\author{
GUO-PING CAO $^{1}$, DAN GUI ${ }^{1}$, LU-DI FU ${ }^{1}$, ZHOU-KE GUO $^{1,2}$ and WEN-JUN FU ${ }^{1}$ \\ ${ }^{1}$ Department of Basic Medical Science, Guangzhou University of Chinese Medicine, Guangzhou, Guangdong 510006; \\ ${ }^{2}$ Department of Neurology and Psychology, Shenzhen Hospital of Chinese Medicine, \\ Shenzhen, Guangdong 518033, P.R. China
}

Received May 12, 2015; Accepted April 8, 2016

DOI: $10.3892 / \mathrm{mmr} .2016 .5382$

\begin{abstract}
Dan-zhi-xiao-yao-san is a Traditional Chinese Medicinal formulation widely used for the treatment of neuropsychological disorders. The present study examined the anxiolytic and neuroprotective effects of Dan-zhi-xiao-yao-san in a rat model of chronic stress. The results of an elevated plus maze test showed that Dan-zhi-xiao-yao-san significantly attenuated the levels of anxiety-induced stress as evidenced by increases in the time spent in the open arm region, as well as the percentage of entries into this area. In addition, Dan-zhi-xiao-yao-san alleviated stress-induced neuronal death, as indicated by histological examination. Furthermore, mechanistic studies suggested that the anxiolytic and neuroprotective effects of Dan-zhi-xiao-yao-san may be mediated via attenuation of chronic stress-induced upregulation of $\alpha$-synuclein and corticosterone, and downregulation of protein phosphatase $2 \mathrm{~A}$ (PP2A) in the hippocampal region of the brain at the mRNA and protein level. In addition, Dan-zhi-xiao-yao-san decreased the serum levels of stress-induced corticosterone in the model animals. In conclusion, the present study demonstrated that Dan-zhi-xiao-yao-san exerted anxiolytic and neuroprotective effects in a rat model of chronic stress via attenuation of stress-induced upregulation of $\alpha$-synuclein and corticosterone, and downregulation of PP2A in the hippocampus.
\end{abstract}

Correspondence to: Dr Zhou-Ke Guo, Department of Neurology and Psychology, Shenzhen Hospital of Chinese Medicine, 1 Fuhua Road, Futian, Shenzhen, Guangdong 518033, P.R. China E-mail: szzyygzk@126.com

Dr Wen-Jun Fu, Department of Basic Medical Science, Guangzhou University of Chinese Medicine, 232 East Waihuan Road, Guangzhou University City, Guangzhou, Guangdong 510006, P.R. China

E-mail: fuqingzhu2006@163.com

Key words: Dan-zhi-xiao-yao-san, anxiolytic, stress, $\alpha$-synuclein, corticosterone

\section{Introduction}

Anxiety-associated disorders constitute the largest class of human psychopathologies and represent a major public health problem $(1,2)$. While the causes of these disorders largely remain elusive, interactions between environmental and genetic factors are thought to be crucial for their pathogenesis and progression $(2,3)$. In addition, various complexities of the central nervous system make anxiety-associated disorders exceptionally difficult to diagnose and treat. At present, serotonin-norepinephrine re-uptake inhibitors, selective serotonin re-uptake inhibitors and pregabalin are the first-line drugs used to clinically treat anxiety-associated disorders $(4,5)$. However, side effects can limit their long-term use (5) and therefore, substantial effort has been made to develop novel drugs to treat anxiety-associated disorders.

Traditional Chinese Medicine (TCM) is widely used for treating a variety of conditions, including anxiety-associated disorders $(6,7)$. Xiao-yao-san and its modified formulations, including Dan-zhi-xiao-yao-san, are most commonly used in China and other Asian countries. According to the theories of TCM, Xiao-yao-san formulations repair the liver, promote liver qi circulation, nourish the blood in the liver and fortify the spleen, and these formulations are used clinically to treat a variety of conditions, including menopausal syndrome, anemia, functional uterine bleeding, hepatitis, chronic gastritis, pelvic inflammatory disease, anxiety and depression (8-10). Dan-zhi-xiao-yao-san is composed of Atractylodis macrocephale rhizoma, Bupleuri radix, Angelicae sinensis, poria, Glycyrrihizae radix, tree peony bark, Gardenia jasminoides, Paeonia lactiflora Pall, mint and roasted ginger. Although Dan-zhi-xiao-yao-san is widely used clinically in China, its underlying mechanisms of action have largely remained elusive. Previous studies have suggested that Dan-zhi-xiao-yao-san prevents dimethylnitrosamine-induced hepatic fibrosis by functioning as an antioxidant (11), and exerts antidepressive effects by modulating neurosteroids $(12,13)$. However, the anxiolytic and neuroprotective effects of Dan-zhi-xiao-yao-san and their underlying molecular mechanisms remain to be determined.

The present study demonstrated the anxiolytic and neuroprotective effects of Dan-zhi-xiao-yao-san in an animal model 
of chronic stress. Furthermore, mechanistic investigation suggested that the mechanism of action of this formulation may involve the modulation of expression levels of protein phosphatase 2A (PP2A), $\alpha$-synuclein and corticosterone.

\section{Materials and methods}

Drugs and chemicals. The commercially available formulation Dan-zhi-xiao-yao-san was obtained from E-FONG Pharmaceutical Corp. (Guangzhou, China). The manufacturer performed quality control testing of individual formulation components to ensure their uniformity. The formulation contained the following 10 medicinal herbs: Atractylodis macrocephale rhizoma, Bupleuri radix, Angelicae sinensis, poria, Glycyrrihizae radix, tree peony bark, Gardenia jasminoides, Paeonia lactiflora Pall, mint and roasted ginger. The powder was dissolved in $0.9 \% \mathrm{NaCl}$ prior to use. Mifepristone was purchased from Sigma-Aldrich (St. Louis, MO, USA). Monoclonal mouse anti- $\alpha$-synuclein (ab27766), polyclonal rabbit anti-PP2A (ab137825) and monoclonal mouse anti-glyceraldehyde-3-phosphate dehydrogenase (GAPDH; ab8245) were obtained from (Abcam, Cambridge, UK). Unless otherwise indicated, all other chemicals were purchased from Sigma-Aldrich.

Animals and drug intervention. Female Sprague-Dawley rats $(\mathrm{n}=50$; aged, 2-3 months; weight, 200-250 g) were bred in the Laboratory Animal Care Facility at Guangzhou University of Chinese Medicine (Guangzhou, China) and maintained under constant conditions of $22 \pm 1^{\circ} \mathrm{C}, 60 \pm 10 \%$ relative humidity and a $12 \mathrm{~h} \mathrm{light/dark} \mathrm{cycle.} \mathrm{All} \mathrm{animal} \mathrm{studies} \mathrm{were} \mathrm{performed} \mathrm{in}$ accordance with international ethical standards, and the study protocols were approved by the AnimalCare and Use Committee of Guangzhou University of Chinese Medicine (Guangzhou, China). The rats were randomly assigned to one of five groups ( $\mathrm{n}=10 /$ group), which included a control group, chronic stress group (Model), chronic stress + low-dose Dan-zhi-xiao-yao-san group (Low; $5.256 \mathrm{~g} / \mathrm{kg}$ Dan-zhi-xiao-yao-san), chronic stress + high-dose Dan-zhi-xiao-yao-san group (High; $52.56 \mathrm{~g} / \mathrm{kg}$ Dan-zhi-xiao-yao-san), and a chronic stress + mifepristone group ( $25 \mathrm{mg} / \mathrm{kg}$ mifepristone as a positive control). The chronic stress model was generated, as previously described with certain modifications (14). Briefly, the rats were subjected to a stress-inducing condition once daily over a period of 21 days. The order of stressor used was as follows: i) Food deprivation for $24 \mathrm{~h}$, ii) water deprivation for $24 \mathrm{~h}$; iii) electric foot shocks for $30 \mathrm{~min}$ (1 mA; duration, $1 \mathrm{sec}$; average frequency, 1 shock/min), iv) forced swimming at $4^{\circ} \mathrm{C}$ for $5 \mathrm{~min}$ and v) immobilization for $6 \mathrm{~h}$. The rats in the drug treatment groups were administered the specified dose of Dan-zhi-xiao-yao-san or mifepristone by intragastric administration each day, $4 \mathrm{~h}$ prior to exposure to the stressful condition, whereas rats in the control group were administered the same volume of distilled water. The rats in all five groups were sacrificed $24 \mathrm{~h}$ after their final exposure to stressful conditions.

Elevated plus maze test. The elevated plus maze test was performed, as previously described (15). Briefly, the elevated plus maze consisted of two closed arms, each $49 \mathrm{~cm}$ long, $10 \mathrm{~cm}$ wide and $30 \mathrm{~cm}$ high, and two open arms. The arms of the apparatus are elevated $50 \mathrm{~cm}$ above the ground. Each rat is placed at the junction of the open and closed arms with the head pointed towards one open arm. The data regarding the rat performance in the maze test were automatically recorded using the Any-maze video tracking system (Stoelting Company; Wood Dale, IL, USA). Any-Maze can be programmed to automatically record all possible indices in each task and digitally records a video of a rat's performance in each test. The number of entries and the duration spent on the open arms are recorded for periods of $5 \mathrm{~min}$. An increase in open arm activity (duration and/or number of entries) reflects anti-anxiety behavior.

Hematoxylin and eosin (HE) staining of tissue sections. The neuroprotective effects of Dan-zhi-xiao-yao-san were examined by observing the histological integrity and damage to tissue in the hippocampus of each rat brain following staining with HE. Briefly, each rat brain was isolated and cut into $5 \mu \mathrm{m}$ sections. The sections were subsequently stained using a combination of Mayer's hematoxylin and $0.5 \%$ aqueous eosin and observed under a FV1000 light microscope (Olympus, Tokyo, Japan). When using this method, the nucleus and other acidic structures in the cells were stained blue, while the cytoplasm was stained red.

Immunohistochemical staining. Expression levels of $\alpha$-synuclein and PP2A in the hippocampus region of the sections of rat midbrain were examined by immunohistochemical staining, as previously described (16). Briefly, the paraffin sections of rat midbrain were deparaffinized and hydrated. The antigenic sites were exposed by incubation in $10 \mathrm{mM}$ citrate buffer $(\mathrm{pH} 6.0)$ at $90^{\circ} \mathrm{C}$, and endogenous peroxidase activity was quenched. Following blocking with $5 \%$ bovine serum albumen in phosphate-buffered saline with Tween-20, the tissue sections were incubated with primary antibodies, followed by incubations with goat anti-rabbit IgG conjugated to horseradish peroxidase (HRP; ab97051, Abcam)and 3,3-N-diaminobenzidine tertrahydrochloride (Solarbio, Beijing, China) to reveal $\alpha$-synuclein and PP2A expression. The sections were subsequently re-stained with HE and observed under a microscope (FV1000; Olympus).

Western blotting analysis. Protein was extracted using radioimmuniprecipitation assay buffer (Beyotime Institute of Biotechnology, Shanghai, China) and centrifuged at $1,000 \mathrm{x} \mathrm{g}$ for $5 \mathrm{~min}$ at $4^{\circ} \mathrm{C}$. The protein concentration in the resulting supernatant was quantified using a bicinchoninic acid assay (Beyotime Institute of Biotechnology). Samples of denatured protein $(20 \mu \mathrm{g})$ were resolved by sodium dodecyl sulfate-polyacrylamide gel electrophoresis and transferred onto a polyvinylidene difluoride membrane (EMD Millipore, Billerica, MA, USA). Following blocking with non-fat milk for $2 \mathrm{~h}$ at room temperature, the membrane was incubated overnight at $4^{\circ} \mathrm{C}$ with antibodies against $\alpha$-synuclein, PP2A, and GAPDH (1:500). Following primary antibody incubation, the membrane was incubated with the goat anti-rabbit HRP-conjugated secondary antibody (ab97051, Abcam) for $1 \mathrm{~h}$ at room temperature. Enhanced chemiluminescence Advance Western blotting detection reagents (GE Healthcare, 
Buckinghamshire, UK) were used for detection. The relative expression of $\alpha$-synuclein and PP2A, relative to GAPDH, were quantified using ImageJ software (National Institutes of Health, Bethesda, MD, USA).

Reverse transcription-quantitative polymerase chain reaction (RT-qPCR). Samples of brain tissue from the hippocampus region were analyzed for the mRNA expression of $\alpha$-synuclein, PP2A and an internal control, $\beta$-actin, by RT-qPCR. Briefly, the total RNA was extracted using TRIzol reagent (Invitrogen; Thermo Fisher Scientific, Inc., Waltham, MA, USA). cDNA synthesis was performed using $2 \mu \mathrm{g}$ RNA and a High-Capacity cDNA Reverse Transcription kit (Thermo Fisher Scientific, Inc.). The mRNA expression of $\alpha$-synuclein, PP2A and $\beta$-actin were detected and amplified by real-time PCR using a Bestar ${ }^{\mathrm{TM}}$ Real time PCR Master Mix kit (Biomart, Beijing, China). The final expression levels were calculated following normalization against the expression of $\beta$-actin. A total of three independent experiments were performed, and each analysis was performed in triplicate. The forward and reverse primers (Sangon Biotech, Shanghai, China) used are shown in Table I.

Determination of corticosterone contents by enzyme-linked immunosorbent assay (ELISA). Plasma samples were analyzed for corticosterone levels using an ELISA kit (cat. no. K014-H1; Arbor Assays, Ann Arbor, MI, USA) and following instructions provided by the manufacturer. All analyses were performed in triplicate.

Statistical analysis. The data are expressed as the mean \pm standard deviation. Statistically significant differences between the groups were identified by one-way analysis of variance, followed by the paired Student's t-test. $\mathrm{P}<0.05$ was considered to indicate a statistically significant difference.

\section{Results}

Antianxiety effect of Dan-zhi-xiao-yao-san. The elevated plus maze test is a behavioral assay widely used to measure the effects of antianxiety agents in rodents (15), and the percentage of time spent in open arms and the number of entries into open arms in this test are sensitive biomarkers for levels of anxiety (15). In the present study, both open arm durations and open arm entries were significantly decreased in the chronic stress rat model (Fig. 1), suggesting that the model can be used to produce an anxiety-like effect. Rats treated with either Dan-zhi-xiao-yao-san or mifepristone for 21 consecutive days by intragastric administration exhibited significantly decreased levels of anxiety as evidenced by their lesser degrees of decrease in open arm durations and open arm entries (Fig. 1), indicating that both Dan-zhi-xiao-yao-san and mifepristone exerted dose-dependent antianxiety effects.

Neuroprotective effect of Dan-zhi-xiao-yao-san. Microscopic examinations of rat mid-brain areas stained with $\mathrm{HE}$ revealed that chronic stress induced degenerative changes in the hippocampus region of stressed rats when compared with non-stressed rats (Fig. 2). Rats in the model group exhibited neuronal cells with pyknotic nuclei, which were clearly distinguishable from viable neuronal cells in the control group that displayed round and pale stained nuclei. However, following intragastric administration of either Dan-zhi-xiao-yao-san or mifepristone for 21 consecutive days, neuronal cell death was significantly inhibited. These results demonstrated that Dan-zhi-xiao-yao-san exerted a neuroprotective effect in animals subjected to chronic stress.

Dan-zhi-xiao-yao-san modulates the expression of $\alpha$-synuclein and PP2A. The effects of our chronic stress model on the expression of $\alpha$-synuclein and PP2A proteins were assessed in the hippocampus region by immunohistochemical methods. As shown in Fig. 3A and B, chronic stress induced increased expression of $\alpha$-synuclein and decreased expression of PP2A, when compared with these expression levels in the control group. Next, the protein expression levels of $\alpha$-synuclein and PP2A were assessed by western blotting. Similar to the immunohistochemical staining, it was revealed that chronic stress significantly upregulated the expression of $\alpha$-synuclein and downregulated the expression of PP2A in the hippocampus region when compared with these expression levels in the control group (Fig. 4). Whether the induced changes in the protein expression levels of $\alpha$-synuclein and PP2A were modulated via expression of their respective mRNAs was next determined. RT-qPCR revealed that exposure to chronic stress induced increased expression of $\alpha$-synuclein and decreased expression of PP2A, by modulating their respective mRNA levels (Fig. 5). The effects of Dan-zhi-xiao-yao-san and mifepristone on chronic stress-induced changes in $\alpha$-synuclein and PP2A were determined by immunohistochemical, western blotting and RT-qPCR methods. As shown in Figs. 3-5, Dan-zhi-xiao-yao-san markedly inhibited chronic stress-induced upregulation of $\alpha$-synuclein. Additionally, Dan-zhi-xiao-yao-san markedly inhibited chronic stress-induced downregulated of PP2A. Finally, as a positive control, mifepristone reversed the chronic stress-induced changes in $\alpha$-synuclein and PP2A expression. Collectively, these results suggested that Dan-zhi-xiao-yao-san modulated changes in the expression levels of $\alpha$-synuclein and PP2A, induced by chronic stress.

Dan-zhi-xiao-yao-san modulates the expression of corticosterone. The present study next investigated the effects of Dan-zhi-xiao-yao-san on chronic stress-induced expression of corticosterone using ELISA to determine the levels of corticosterone in rat serum. As shown in Fig. 6, the levels of corticosterone in serum samples from the model group were significantly higher compared with those in the control group. By contrast, both Dan-zhi-xiao-yao-san and mifepristone significantly reversed the chronic stress-induced changes in corticosterone levels.

\section{Discussion}

Dan-zhi-xiao-yao-san is one of several herbal medicines commonly used for treating various diseases in China, including anxiety-associated diseases, including menopausal syndrome, insomnia and depression (17-19). However, the underlying mechanisms for its clinical effects are poorly understood. In the present study, Dan-zhi-xiao-yao-san exhibited antianxiety 
Table I. Primers used in the present study.

\begin{tabular}{|c|c|c|}
\hline Gene & GenBank accession no. & Primer sequence $\left(5^{\prime} \rightarrow 3^{\prime}\right)$ \\
\hline$\alpha$-Synuclein & NM_019169.2 & $\begin{array}{l}\text { F: CCCACAAGAGGGAATCCTGG } \\
\text { R: AAGCCTCACTGCTAGGGTCC }\end{array}$ \\
\hline $\mathrm{PP} 2 \mathrm{~A}$ & NM_053999.2 & $\begin{array}{l}\text { F: GAGGCGAGCCACATGTCACT } \\
\text { R: CCATTAGGTCAACAGACGGTGTT }\end{array}$ \\
\hline$\beta$-actin & NM_031144.3 & $\begin{array}{l}\text { F: GGAGATTACTGCCCTGGCTCCTA } \\
\text { R:GACTCATCGTACTCCTGCTTGCTG }\end{array}$ \\
\hline
\end{tabular}

F, forward; R, reverse; PP2A, protein phosphatase 2A.

and neuroprotective effects in a rat model of chronic stress. It was demonstrated that Dan-zhi-xiao-yao-san significantly inhibited stress-induced upregulation of $\alpha$-synuclein and downregulation of $\mathrm{PP} 2 \mathrm{~A}$ in the hippocampus region of rat brains. Additionally, Dan-zhi-xiao-yao-san also significantly attenuated stress-induced upregulation of corticosterone. It was hypothesized that these findings may also explain the clinical effects of Dan-zhi-xiao-yao-san in patients with anxiety-associated diseases. It was noted that the antianxiety effects of Dan-zhi-xiao-yao-san were comparable to those shown by mifepristone, suggesting that Dan-zhi-xiao-yao-san and traditional anxiolytics, including mifepristone, may exhibit similar clinical efficacies.

When exposed to conditions of stress, the body makes adaptive changes to maintain homeostasis. However, high intensity stressors may disrupt the body's adaptive abilities (20), resulting in organ damage and disease. In fact, stress has an important role in the pathophysiology of several neurological diseases, including Parkinson's disease, Alzheimer's disease, post-traumatic stress disorder, depression and schizophrenia (20-22). In addition, increasing evidence indicates that stressful experiences, which occur throughout life, are crucial for the development and pathogenesis of several psychiatric disorders, including anxiety and depression (23). In animal models, chronic stressors cause behavioral changes associated with anxiety disorders $(24,25)$; for example, foot shock, exposure to cold water and immobilization have been reported to induce anxiety-like effects in rodents $(14,26)$.

In our animal model, stress was produced by exposing rats to a stress-inducing condition (either electric foot shocks, swimming in ice-cold water or immobilization) once daily, at randomly selected times, for 21 consecutive days. The present study subsequently used the elevated plus maze test to determine whether the rats exhibited anxiety-associated symptoms and behaviors. When using the elevated plus maze test, the percentage of time spent by a rat in open arms and the number of open arm entries are sensitive biomarkers for anxiety (15). As shown in Fig. 1, the open arm activity (duration and entries) by rats exposed to our chronic stress model were significantly decreased, suggesting that the stress model caused anxiety-like effects in rats. Additionally, this chronic stress model was used to test the antianxiety effects of Dan-zhi-xiao-yao-san. The results revealed that Dan-zhi-xiao-yao-san significantly attenuated chronic stress-induced anxiety in a dose-dependent manner, when compared with results shown by control rats.
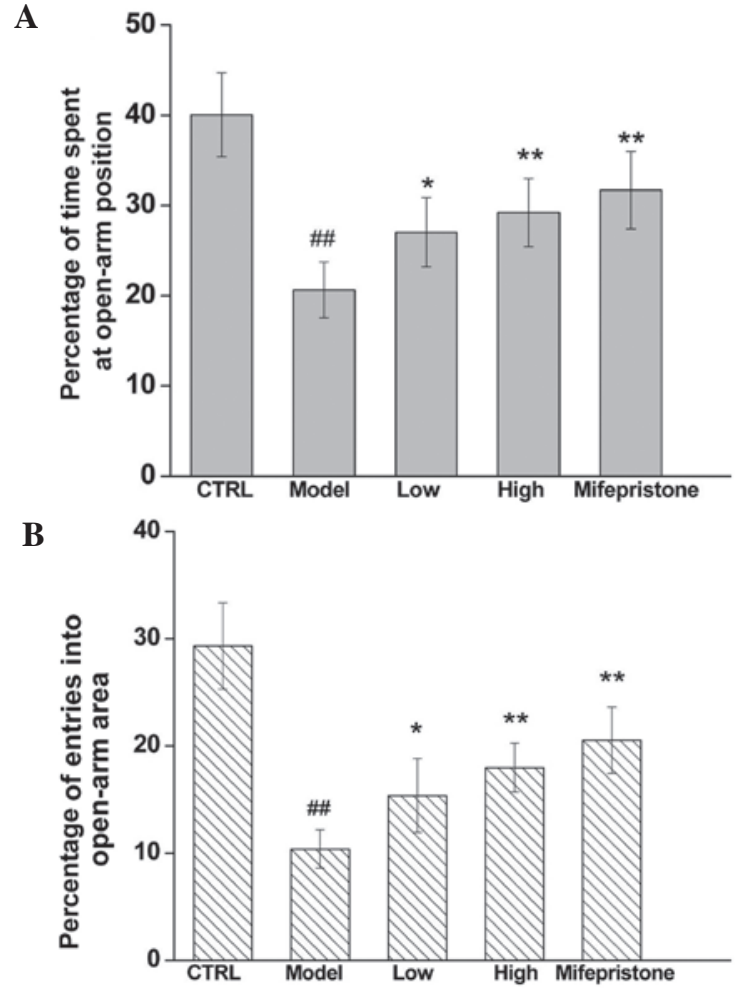

Figure 1. Anxiolytic effects of Dan-zhi-xiao-yao-san in the elevated plus maze test. The effects of Dan-zhi-xiao-yao-san on (A) open arm duration and (B) open arm entries of model rats with induced chronic stress in the elevated plus maze test. The data are expressed as the mean \pm standard deviation $\left({ }^{\# \#} \mathrm{P}<0.01\right.$ vs. CTRL; ${ }^{*} \mathrm{P}<0.05,{ }^{* *} \mathrm{P}<0.01$ vs. chronic stress model group). CTRL, normal rats; Model, rats with induced chronic stress; Low, model rats treated with $5.256 \mathrm{~g} / \mathrm{kg}$ Dan-zhi-xiao-yao-san; High, model rats treated with $52.56 \mathrm{~g} / \mathrm{kg}$ Dan-zhi-xiao-yao-san; Mifepristone, model rats treated with $25 \mathrm{mg} / \mathrm{kg}$ mifepristone.

Mifepristone is a well established antianxiety agent (27), and in the present study, this compound was used as a positive control to assist with confirming the pharmacological effects of Dan-zhi-xiao-yao-san. It was revealed that a high dose of Dan-zhi-xiao-yao-san produced effects similar to those produced by mifepristone in the elevated plus maze test, further confirming the marked antianxiety effect of Dan-zhi-xiao-yao-san.

Several neurodegenerative diseases, including Parkinson's and Alzheimer's disease, are accompanied by neuronal death and feelings of anxiety $(28,29)$. Previous studies with rats have shown that extreme chronic stress may cause neuronal 

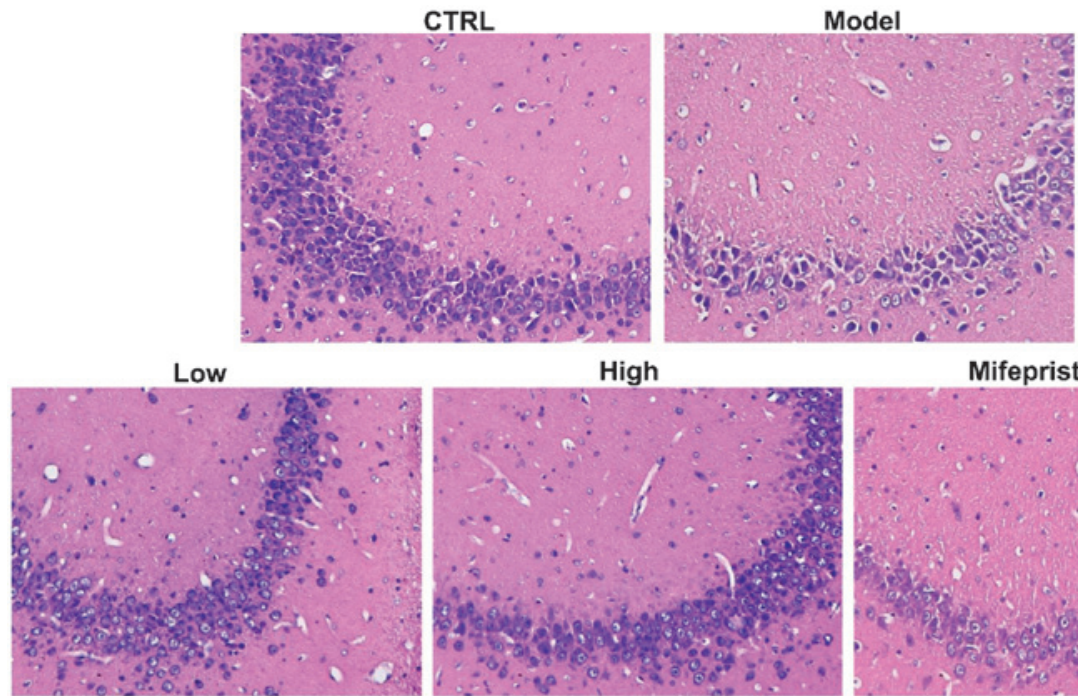

High

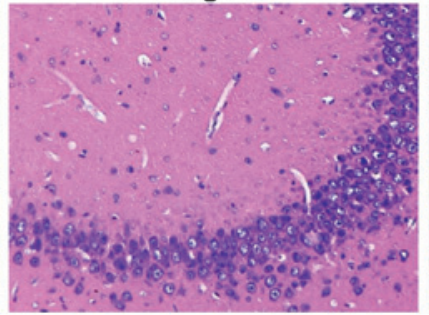

Mifepristone

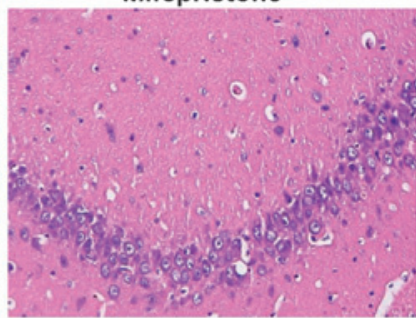

Figure 2. Neuroprotective effects of Dan-zhi-xiao-yao-san on rats with induced chronic stress. Following treatment with Dan-zhi-xiao-yao-san, mifepristone or vehicle control, hematoxylin and eosin staining was performed to observe histological integrity and tissue damage in the hippocampal region of rat brains (magnification, x200). CTRL, normal rats; Model, rats with induced chronic stress; Low, model rats treated with 5.256 g/kg Dan-zhi-xiao-yao-san; High, model rats treated with $52.56 \mathrm{~g} / \mathrm{kg}$ Dan-zhi-xiao-yao-san; Mifepristone, model rats treated with $25 \mathrm{mg} / \mathrm{kg}$ mifepristone.

A
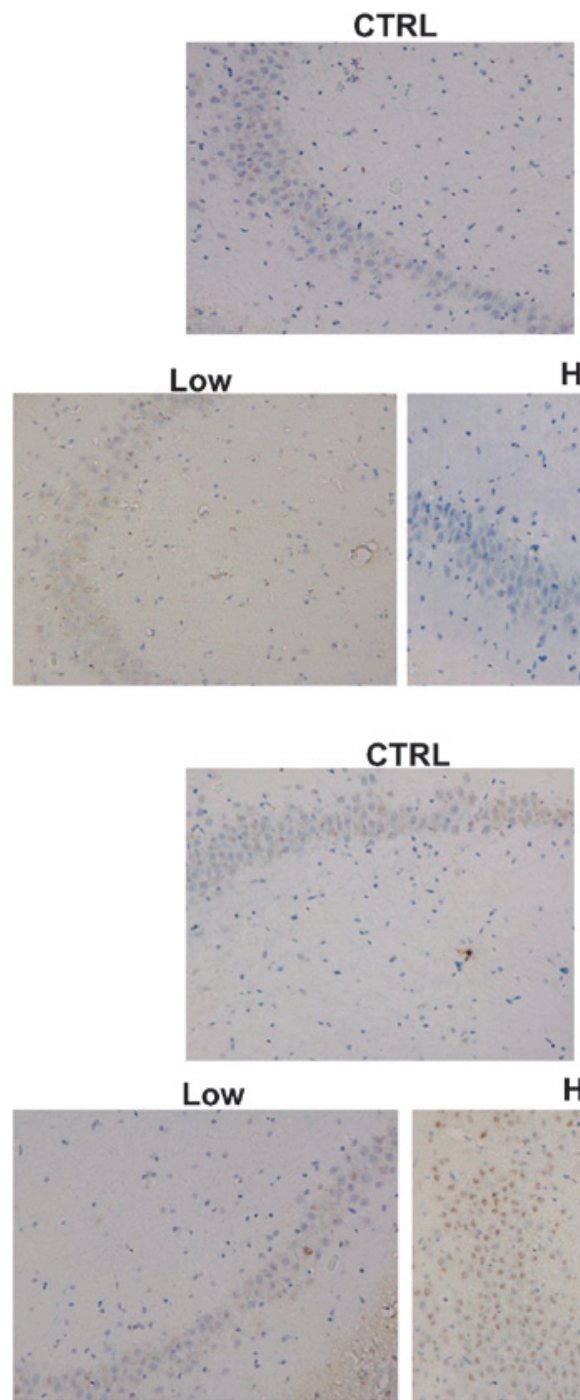

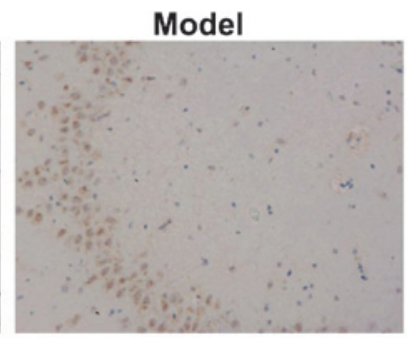

High

Mifepristone

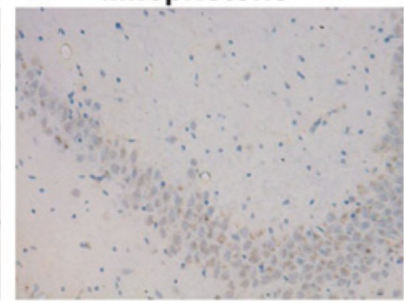

Model

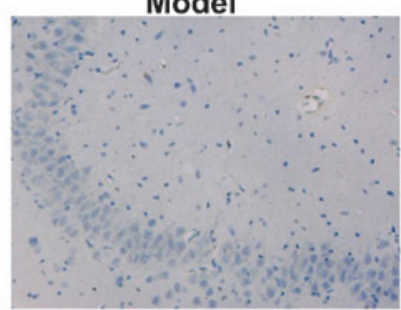

High

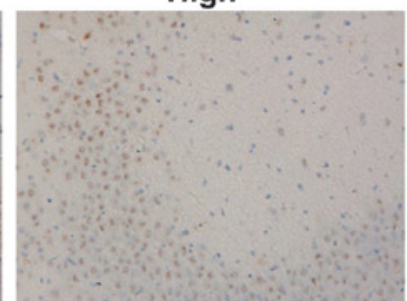

Mifepristone

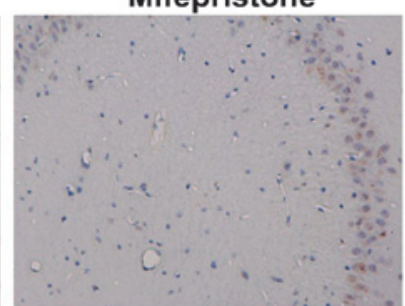

Figure 3. Effects of Dan-zhi-xiao-yao-san on the expression levels of (A) $\alpha$-synuclein and (B) phosphatase 2A in the hippocampal regions of rats with induced chronic stress were detected by immunochemical staining analysis (magnification, x200). CTRL, normal rats; Model, rats with induced chronic stress; Low, model rats treated with $5.256 \mathrm{~g} / \mathrm{kg}$ Dan-zhi-xiao-yao-san; High, model rats treated with $52.56 \mathrm{~g} / \mathrm{kg}$ Dan-zhi-xiao-yao-san; Mifepristone, model rats treated with $25 \mathrm{mg} / \mathrm{kg}$ mifepristone. 

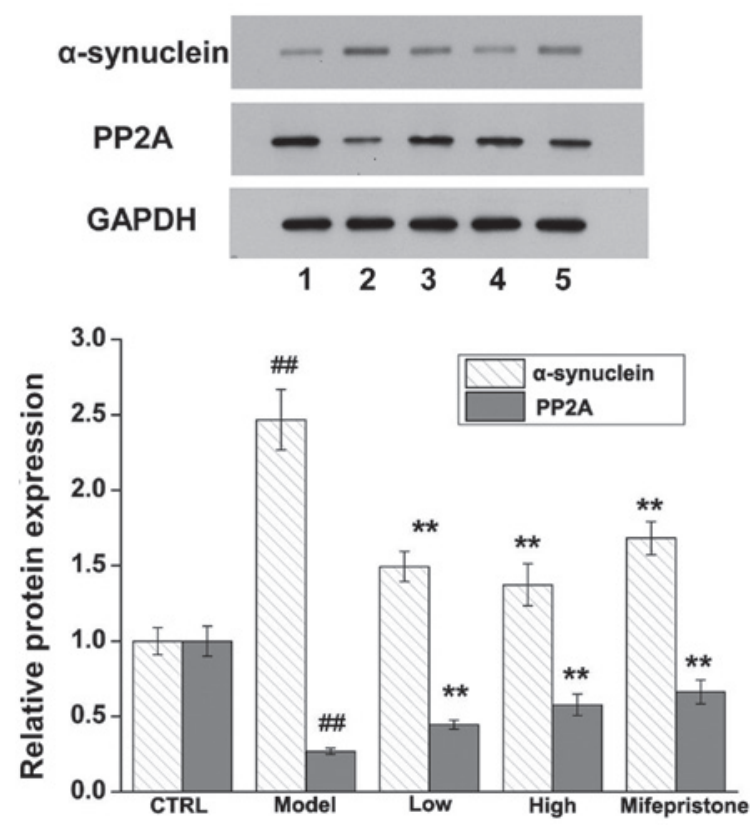

Figure 4. Dan-zhi-xiao-yao-san reduces stress-induced overexpression of $\alpha$-synuclein and downregulation of PP2A in the hippocampal region. Western blotting was performed to detect the protein expression levels of $\alpha$-synuclein and PP2A in the various groups. Protein expression was quantified by densitometric analysis of bands. Lanes 1-5 correspond to CTRL, Model, Low, High and Mifepristone, respectively. The data are expressed as the mean \pm standard deviation $\left(\mathrm{n}=3 ;{ }^{\# \#} \mathrm{P}<0.01\right.$ vs. CTRL; ${ }^{* *} \mathrm{P}<0.01$ vs. chronic stress model group). CTRL, normal rats; Model, rats with induced chronic stress; Low, model rats treated with $5.256 \mathrm{~g} / \mathrm{kg}$ Dan-zhi-xiao-yao-san; High, model rats treated with $52.56 \mathrm{~g} / \mathrm{kg}$ Dan-zhi-xiao-yao-san; Mifepristone, model rats treated with $25 \mathrm{mg} / \mathrm{kg}$ mifepristone.

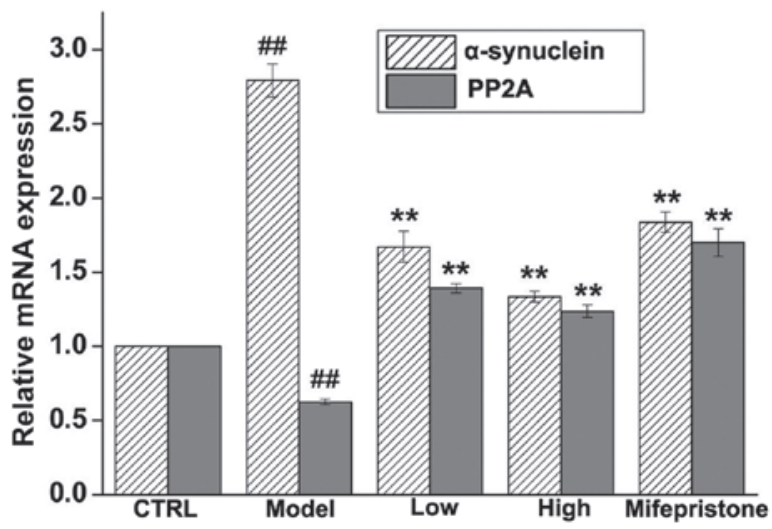

Figure 5. Dan-zhi-xiao-yao-san reduces stress-induced mRNA overexpression of $\alpha$-synuclein and downregulation of PP2A in the hippocampal region of rats. Reverse-transcription-quantitative polymerase chain reaction was performed to detect changes in the mRNA expression levels of $\alpha$-synuclein and PP2A in the various groups. The data are expressed as the mean \pm standard deviation $\left(\mathrm{n}=3 ;{ }^{\# \#} \mathrm{P}<0.01\right.$ vs. CTRL; ${ }^{* *} \mathrm{P}<0.01$ vs. chronic stress model group). CTRL, normal rats; Model, rats with induced chronic stress; Low, model rats treated with $5.256 \mathrm{~g} / \mathrm{kg}$ Dan-zhi-xiao-yao-san; High, model rats treated with $52.56 \mathrm{~g} / \mathrm{kg}$ Dan-zhi-xiao-yao-san; Mifepristone, model rats treated with $25 \mathrm{mg} / \mathrm{kg}$ mifepristone.

injuries, which selectively affect hippocampus structure (30). Therefore, in the present study, HE staining was used to investigate the effects of Dan-zhi-xiao-yao-san on stress-induced neuronal death, and particularly in the hippocampus region. Rats exposed to chronic stress exhibited hippocampus neurons

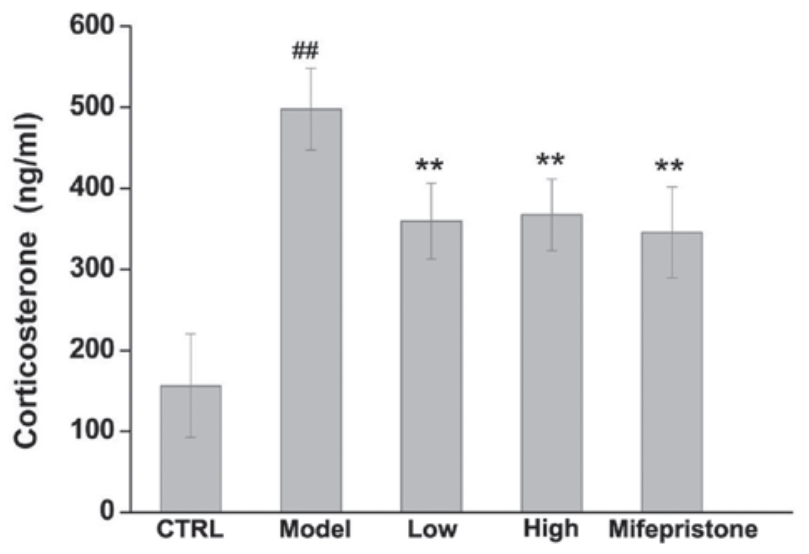

Figure 6. Effects of Dan-zhi-xiao-yao-san on serum corticosterone levels in rats subjected to chronic stress. An enzyme-linked immunosorbent assay was performed to assess the serum levels of corticosterone. The data are expressed as the mean \pm standard deviation $\left(\mathrm{n}=3 ;{ }^{\# \#} \mathrm{P}<0.01\right.$ vs. CTRL; ${ }^{* *} \mathrm{P}<0.01$ vs. chronic stress model group). CTRL, normal rats; Model, rats with induced chronic stress; Low, model rats treated with $5.256 \mathrm{~g} / \mathrm{kg}$ Dan-zhi-xiao-yao-san; High, model rats treated with $52.56 \mathrm{~g} / \mathrm{kg}$ Dan-zhi-xiao-yao-san; Mifepristone, model rats treated with $25 \mathrm{mg} / \mathrm{kg}$ mifepristone.

with pyknotic nuclei, indicative of neuronal death, while rats in the Dan-zhi-xiao-yao-san-treated groups displayed viable neurons with round and pale stained nuclei. These results indicated that Dan-zhi-xiao-yao-san attenuated stress-induced neuronal death. Additionally, the positive control compound, mifepristone, also attenuated stress-induced neuronal death in the hippocampus region.

After finding that Dan-zhi-xiao-yao-san exerted marked antianxiety and neuroprotective effects, the present study examined the possible mechanisms for those effects. $\alpha$-synuclein, the predominant component of Lewy bodies, serves an important role in the pathogenesis of Parkinson's and several other diseases (31). In addition, anxiety is a well-known feature of anxiety-associated diseases, including Parkinson's disease (32), and it is believed that $\alpha$-synuclein may be important in anxiety. Results of previous studies have suggested that a genomic region located close to the $\alpha$-synuclein gene may be associated with anxiety-associated behaviors in animals (33). Additionally, the levels of $\alpha$-synuclein are known to be elevated in the hippocampus of innate anxiety rats (34). In the present study, immunohistochemistry, western blotting and RT-qPCR were used to show that exposure to chronic stress upregulated the mRNA and protein expression levels of $\alpha$-synuclein in the rat hippocampus region. However, treatment with either Dan-zhi-xiao-yao-san or mifepristone significantly inhibited these stress-induced increases. Furthermore, Dan-zhi-xiao-yao-san inhibited the expression of $\alpha$-synuclein in a dose-dependent manner, and a high dose of Dan-zhi-xiao-yao-san was more effective than mifepristone for downregulating the expression of $\alpha$-synuclein. PP2A is important for the regulation of MAP kinases, including extracellular signal-regulated kinases $1 / 2$, c-Jun N-terminal kinases and p38 (35), and also serves important roles in regulating tyrosine hydroxylase synthesis and $\alpha$-synuclein expression (36). In addition, $\alpha$-synuclein reduces the expression and activity of PP2A (37). In the present study, it was revealed that exposure to chronic stress significantly decreased PP2A expression, 
whereas Dan-zhi-xiao-yao-san and mifepristone reversed this effect at both the mRNA and protein levels. The neuroprotective effects of Dan-zhi-xiao-yao-san may be partially attributable to its regulation of the expression levels of $\alpha$-synuclein and PP2A. Taken together, these results showed that the antianxiety and neuroprotective effects of Dan-zhi-xiao-yao-san may involve regulation of $\alpha$-synuclein and PP2A expression.

Finally, the effects of Dan-zhi-xiao-yao-san on corticosterone expression in the rat model of chronic stress were assessed. Corticosterone is the predominant glucocorticoid produced in the cortex of rodent adrenal glands (38). It is well established that conditions which produce stress and anxiety induce expression of corticosterone, which subsequently induces anxiety (39-41). It was revealed that rats in our chronic stress model exhibited high levels of corticosterone in their blood serum (Fig. 6). By contrast, treatment with either Dan-zhi-xiao-yao-san or mifepristone significantly decreased these stress-induced elevated levels of corticosterone.

In conclusion, the present results showed that Dan-zhi-xiao-yao-san had both antianxiety and neuroprotective effects in the rat model of chronic stress. The underlying mechanisms for these effects may involve regulation of $\alpha$-synuclein and PP2A expression, as well as modulation of corticosterone expression. The present study assists with elucidating possible mechanisms for the antianxiety effect of Dan-zhi-xiao-yao-san, and may provide evidence supporting the clinical use of this formulation. However, the individual herbs and other predominant components responsible for the antianxiety and neuroprotective effects of Dan-zhi-xiao-yao-san remain to be clearly identified, and the molecular pathways and underlying mechanisms responsible for those effects require further study.

\section{Acknowledgements}

The present study was supported by the National Natural Science foundation of China (no. 81403304), the Shenzhen Municipal Science and Technology Innovation Council (no. JCYJ20130328154910812) and the Xinhuo Program in Guangzhou University of Chinese Medicine (no. XH20140106). The authors would like to thank Professor Zhi-yu Wang (Guangzhou University of Chinese Medicine, Guangzhou, China) for english language editing of the manuscript.

\section{References}

1. Demyttenaere K, Bruffaerts R, Posada-Villa J, Gasquet I, Kovess V, Lepine JP, Angermeyer MC, Bernert S, de Girolamo G, Morosini P, et al: Prevalence, severity, and unmet need for treatment of mental disorders in the World Health Organization World Mental Health Surveys. JAMA 291: 2581-2590, 2004.

2. Hettema JM, Neale MC and Kendler KS: A review and meta-analysis of the genetic epidemiology of anxiety disorders. Am J Psychiatry 158: 1568-1578, 2001.

3. Sachser N and Lesch KP: The interplay of genotype and environment in the development of fear and anxiety. e-Neuroforum 4 57-62, 2013.

4. Bandelow B, Sher L, Bunevicius R, Hollander E, Kasper S, Zohar J and Möller HJ; WFSBP Task Force on Mental Disorders in Primary Care; WFSBP Task Force on Anxiety Disorders, OCD and PTSD: Guidelines for the pharmacological treatment of anxiety disorders, obsessive-compulsive disorder and posttraumatic stress disorder in primary care. Int J Psychiatry Clin Pract 16: 77-84, 2012.
5. Farach FJ, Pruitt LD, Jun JJ, Jerud AB, Zoellner LA and Roy-Byrne PP: Pharmacological treatment of anxiety disorders: Current treatments and future directions. J Anxiety Disorders 26: 833-843, 2012.

6. Bystritsky A, Hovav S, Sherbourne C, Stein MB, Rose RD Campbell-Sills L, Golinelli D, Sullivan G, Craske MG and Roy-Byrne PP: Use of complementary and alternative medicine in a large sample of anxiety patients. Psychosomatics 53: 266-272, 2012

7. Sarris J, McIntyre E and Camfield DA: Plant-based medicines for anxiety disorders, part 2: A review of clinical studies with supporting preclinical evidence. CNS Drugs 27: 301-319, 2013.

8. Chen WF, Xu L, Yu CH, Ho CK, Wu K, Leung GC and Wong MS: The in vivo therapeutic effect of free wanderer powder (xiāo yáo săn, Xiaoyaosan) on mice with 4T1 cell induced breast cancer model. J Tradit Complement Med 2: 67-75, 2012.

9. Qin F, Wu XA, Tang Y, Huang Q, Zhang ZJ and Yuan JH: Meta-analysis of randomized controlled trials to assess the effectiveness and safety of free and easy wanderer plus, a polyherbal preparation for depressive disorders. J Psychiatr Res 45: 1518-1524, 2011.

10. Fratkin JP and Dharmananda S (eds): Chinese herbal patent medicines: The clinical desk reference. Shya Publications, Boulder, CO, 2001.

11. Chien SC, Chang WC, Lin PH, Chang WP, Hsu SC, Chang JC, Wu YC, Pei JK and Lin CH: A Chinese herbal medicine, Jia-Wei-Xiao-Yao-San, prevents dimethylnitrosamine-induced hepatic fibrosis in rats. Scientific World Journal 2014: 217525, 2014.

12. Zhang Y, Han M, Liu Z, Wang J, He Q and Liu J: Chinese herbal formula Xiao yao san for treatment of depression: A systematic review of randomized controlled trials. Evid Based Complement and Alternat Med 2012: 931636, 2012.

13. Mizowaki M, Toriizuka K and Hanawa T: Anxiolytic effect of Kami-Shoyo-San (TJ-24) in mice: Possible mediation of neurosteroid synthesis. Life Sci 69: 2167-2177, 2001.

14. Campos AC, Fogaca MV, Aguiar DC and Guimarães FS: Animal models of anxiety disorders and stress. Rev Bras Psiquiatr 35 (Suppl 2): S101-S111, 2013.

15. Walf AA and Frye CA: The use of the elevated plus maze as an assay of anxiety-related behavior in rodents. Nat Protoc 2: 322-328, 2007

16. Volk H, Potschka H and Löscher W: Immunohistochemical localization of P-glycoprotein in rat brain and detection of its increased expression by seizures are sensitive to fixation and staining variables. J Histochem Cytochem 53: 517-531, 2005.

17. Chen HY, Lin YH, Wu JC, Chen YC, Yang SH, Chen JL and Chen TJ: Prescription patterns of Chinese herbal products for menopausal syndrome: Analysis of a nationwide prescription database. J Ethnopharmacol 137: 1261-1266, 2011.

18. Yeung WF, Chung KF, Poon MM, Ho FY, Zhang SP, Zhang ZJ Ziea ET and Wong Taam V: Prescription of chinese herbal medicine and selection of acupoints in pattern-based traditional chinese medicine treatment for insomnia: A systematic review. Evid Based Complement Alternat Med 2012: 902578, 2012.

19. Yeung WF, Chung KF, Ng KY, Yu YM, Ziea ET and Ng BF: A systematic review on the efficacy, safety and types of Chinese herbal medicine for depression. J Psychiatr Res 57: 165-175, 2014.

20. McEwen BS: Central effects of stress hormones in health and disease: Understanding the protective and damaging effects of stress and stress mediators. Eur J Pharmacol 583: 174-185, 2008.

21. Saavedra JM, Sánchez-Lemus E and Benicky J: Blockade of brain angiotensin II A T1 receptors ameliorates stress, anxiety, brain inflammation and ischemia: Therapeutic implications. Psychoneuroendocrinology 36: 1-18, 2011.

22. Djamshidian A and Lees AJ: Can stress trigger Parkinson's disease? J Neurosurg Psychiatry 85: 878-881, 2014.

23. Heim $\mathrm{C}$ and Nemeroff CB: The impact of early adverse experiences on brain systems involved in the pathophysiology of anxiety and affective disorders. Biol Psychiatry 46: 1509-1522, 1999.

24. Adriaan Bouwknecht J, Olivier B and Paylor RE: The stress-induced hyperthermia paradigm as a physiological animal model for anxiety: A review of pharmacological and genetic studies in the mouse. Neurosci Biobehav Rev 31: 41-59, 2007.

25. Haller J and Alicki M: Current animal models of anxiety, anxiety disorders, and anxiolytic drugs. Curr Opin Psychiatry 25: 59-64, 2012.

26. Jaggi AS, Bhatia N, Kumar N, Singh N, Anand P and Dhawan R: A review on animal models for screening potential anti-stress agents. Neurol Sci 32: 993-1005, 2011. 
27. DeBattista $\mathrm{C}$ and Belanoff J: The use of mifepristone in the treatment of neuropsychiatric disorders. Trends Endocrinol Metab 17: 117-121, 2006.

28. Leentjens AF, Dujardin K, Marsh L, Martinez-Martin P, Richard IH and Starkstein SE: Anxiety and motor fluctuations in Parkinson's disease: A cross-sectional observational study. Parkinsonism Relat Disord 18: 1084-1088, 2012.

29. Gallagher D, Coen R, Kilroy D, Belinski K, Bruce I, Coakley D, Walsh B, Cunningham C and Lawlor BA: Anxiety and behavioural disturbance as markers of prodromal Alzheimer's disease in patients with mild cognitive impairment. Int J Geriatr Psychiatry 26: 166-172, 2011.

30. Radley JJ and Morrison JH: Repeated stress and structural plasticity in the brain. Ageing Res Rev 4: 271-287, 2005.

31. Goedert M: Alpha-synuclein and neurodegenerative diseases. Nat Rev Neurosci 2: 492-501, 2001

32. Morris EP, Stewart SH and Ham LS: The relationship between social anxiety disorder and alcohol use disorders: A critical review. Clin Psychol Rev 25: 734-760, 2005.

33. Ramos A, Moisan MP, Chaouloff F, Mormede C and Mormède P: Identification of female-specific QTLs affecting an emotionality-related behavior in rats. Mol Psychiatry 4: 453-462, 1999.

34. Chiavegatto S, Izidio GS, Mendes-Lana A, Aneas I, Freitas TA, Torrão AS, Conceição IM, Britto LR and Ramos A: Expression of alpha-synuclein is increased in the hippocampus of rats with high levels of innate anxiety. Mol Psychiatry 14: 894-905, 2008.
35. Liu Y, Shepherd EG and Nelin LD: MAPK phosphatases-regulating the immune response. Nat Rev Immunol 7: 202-212, 2007.

36. Toska K, Kleppe R, Armstrong CG, Morrice NA, Cohen P and Haavik J: Regulation of tyrosine hydroxylase by stress-activated protein kinases. J Neurochem 83: 775-783, 2002.

37. Wu J, Lou H, Alerte TN, Stachowski EK, Chen J, Singleton AB Hamilton RL and Perez RG: Lewy-like aggregation of $\alpha$-synuclein reduces protein phosphatase $2 \mathrm{~A}$ activity in vitro and invivo. Neuroscience 207: 288-297, 2012.

38. Wittmann W, Schunk E, Rosskothen I, Gaburro S, Singewald N, Herzog H and Schwarzer C: Prodynorphin-derived peptides are critical modulators of anxiety and regulate neurochemistry and corticosterone. Neuropsychopharmacology 34: 775-785, 2009.

39. Shepard JD, Barron KW and Myers DA: Corticosterone delivery to the amygdala increases corticotropin-releasing factor mRNA in the central amygdaloid nucleus and anxiety-like behavior. Brain Res 861: 288-295, 2000.

40. Vallée M, Mayo W, Dellu F, Le Moal M, Simon H and Maccari S: Prenatal stress induces high anxiety and postnatal handling induces low anxiety in adult offspring: Correlation with stress-induced corticosterone secretion. J Neurosci 17: 2626-2636, 1997.

41. Mitra R and Sapolsky RM: Acute corticosterone treatment is sufficient to induce anxiety and amygdaloid dendritic hypertrophy. Proc Nat Acad Sci 105: 5573-5578, 2008. 